\title{
Design of pneumatic manipulator based on motion controller
}

\author{
Gui-Zhi ZHAO ${ }^{1, a}$, Da-Peng LIU ${ }^{2, b}$ \\ ${ }^{1}$ Inner Mongolia University for the Nationalities, College of Mechanical Engineering, Tong Liao, \\ 028000, China; \\ ${ }^{2}$ NO.724 Research Institute under CSIC, Nanjing Jiangsu 210003, China; \\ aguizhi_84@yahoo.com.cn, 'liudapeng@163.com,
}

Keywords: Pneumatic manipulator, Automatic feeding, Control system.

\begin{abstract}
The combination of pneumatic technology and automatic control technology has greatly improved the pneumatic automation technology, expanding the scope of application of pneumatic components. The improvement of industrial automation makes that the application of manipulator is used more widely. A kind of pneumatic manipulator is designed based $i$ on the motion controller in this paper, the manipulator control has high precision, it is stable and reliable, and it has automatic and manual two kinds of mode, it can satisfy different working conditions. It provides a new idea for the development of manipulator.
\end{abstract}

\section{Introduction}

With the continuous development of science and technology, the application of pneumatic technology is used more and more widely in various fields, especially it is the rapid development in the automatic production line [1]. Emergence of motion control technology makes pneumatic technology have a new development, combination of motion control technology and pneumatic technology can improve the automation level of the system, and make the control system more flexible and more reliable [2]. Pneumatic manipulator as a kind of manipulator, it has the advantages of simple structure, stable movement, energy saving and environmental protection and many others, it has a wide range of applications in the field of automatic control [3]. The improvement of industrial automation, it has become inevitable that manipulator replaces human labor. The traditional manipulator is controlled by PLC, but PLC control may exist the problem such as lagging, the control precision will be influenced [4]. In recent years, the motion controller has been widely used with high control precision, fast response and so on [5]. Based on the motion controller as the control core, a kind of pneumatic manipulator is designed based on the motion controller, and it provides a new idea for the development of manipulator.

\section{Overall design of manipulator}

The structure of manipulator. The main function of the pneumatic manipulator is to grasp the workpiece from the starting place, and transport to the designated location steady. As shown in Figure 1 , the manipulator is mainly composed of foundation, motors, cylinders and sucker etc. The motor is used to control rotating motion of lift cylinder and telescopic cylinder, through the rotation of the motor and the movement of the cylinder, the sucker is moved to the specified position, it picks up the workpiece, the workpiece is placed to the specified position by the rotation of the motor and the movement of the cylinder. 


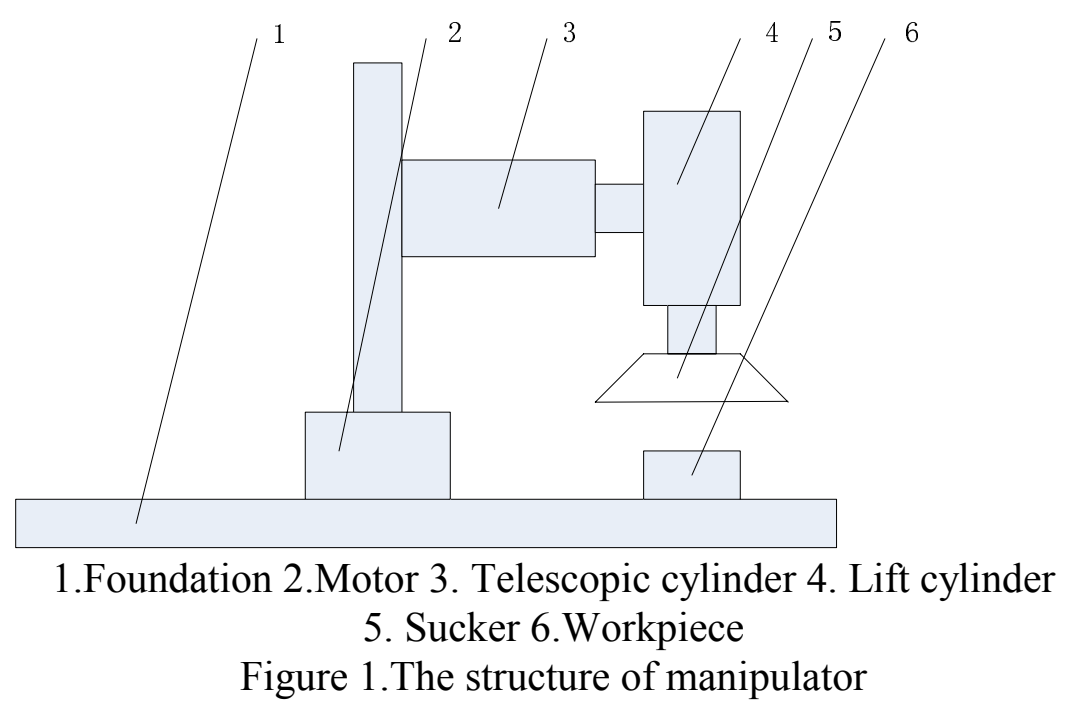

The function of manipulator. The manipulator has two operating modes, respectively they are automatic mode and manual mode. In the manual operation mode, each time the button is pressed, the manipulator move one step, it can realize the manipulator rotation, stretching, lifting, suction and discharge control. In the automatic mode, just press the button once, the manipulator will do rotation, stretching, lifting, suction and discharge control in accordance with the set path, and repeat suction feeding process. In the automatic cycle, you can press the stop button, the manipulator will stop moving.

The design of system. As shown in Figure 2, the pneumatic manipulator uses motion controller as control core, using the motion controller can guarantee the processing speed of the system, and ensure the control precision. The communication between motion controller and PC uses Ethernet. Sensor is installed on the manipulator, the signal is detected by using the sensor, the sensor signal feedback to the system, the system according to the motion program controls the motor and cylinder motion, to ensure accurate operation of manipulator.

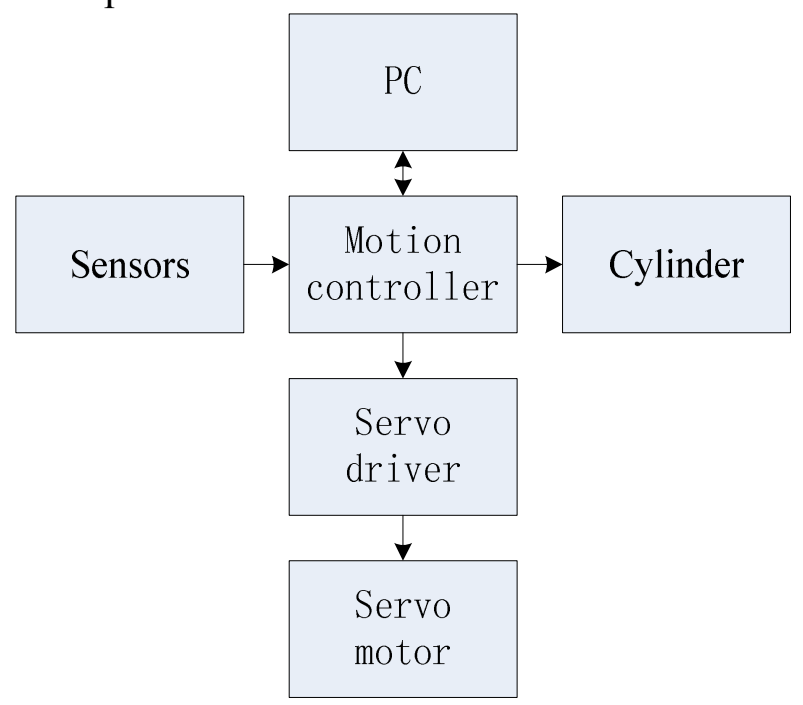

Figure 2. The design of system

\section{Software design}

Software structure design. The core of the control system in this paper is the motion controller, motion controller does not have the program development environment, it needs PC as upper computer, so the software structure uses the form of upper and lower computer. As shown in Figure 3, PC serves as the host machine, it mainly used for program development and management; motion controller as slave machine is mainly used to control the pneumatic mechanical manipulator. As 
shown in Figure 4, the software adopts modular design, modular design can improve the stability, flexibility and portability of the system, it is divided into management module, control module and feedback module. The grasping completes work through the direct coordination of various modules.

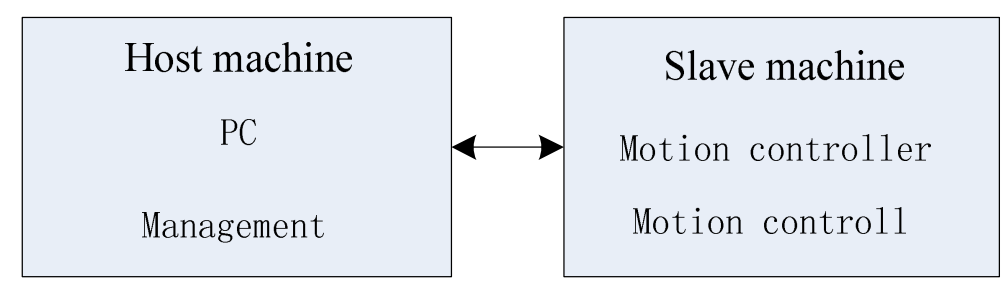

Figure3. The structure design of software

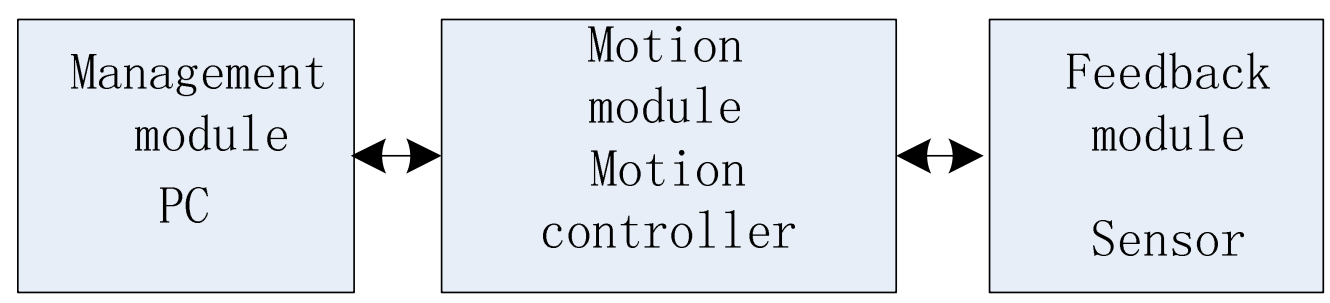

Figure4. The modular design of software

Program flow design. The program flow of system is shown in figure 5. First, system does initialization, detect the state of system, if the system is working properly, then entry the choice mode, a mode selection option is on the system of man-machine interface, if you choose the manual mode you can manually control the manipulator, each button click will perform the corresponding action. If you choose the automatic mode, first it need to design the path of the manipulator, press the button after the manipulator automatic cycle work, if there are any problems, it will return to automatically initialized state. The operator can also stop the movement of the manipulator at any time.

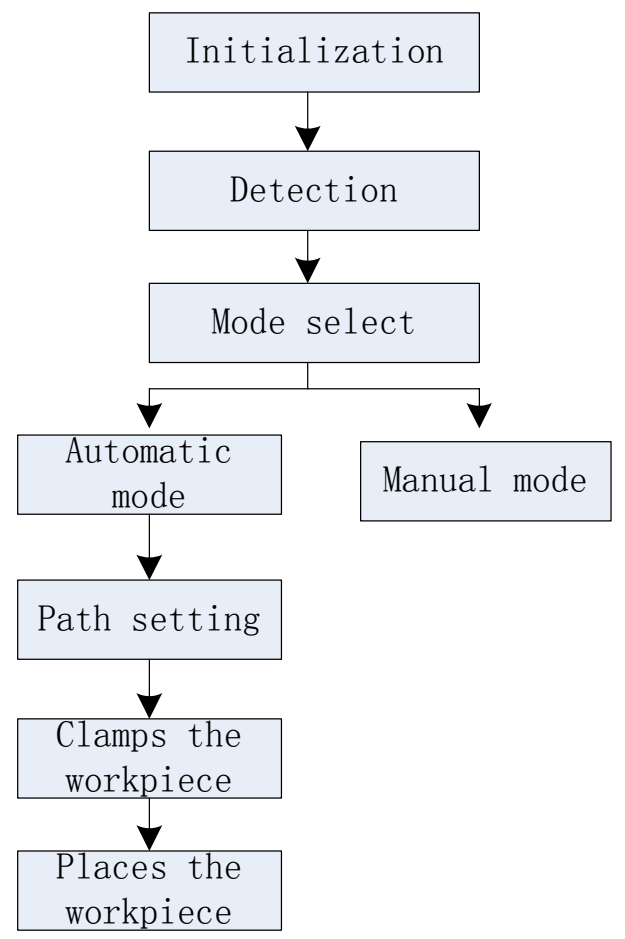

Figure5. Work flow 
I/O design. There are the $\mathrm{I} / \mathrm{O}$ interfaces in the motion controller, each sensor and the motion controller is connected to the control system through $\mathrm{I} / \mathrm{O}$ interface, and it receives and sends signals through I/O interface controller. The design of I/O system is shown in table 1.

Table1. System I/O design

\begin{tabular}{cccc}
\hline & Input & \multicolumn{2}{c}{ Output } \\
\hline IN0 & Position signal 1 & OUT0 & Indicator 1 \\
IN1 & Position signal 2 & OUT1 & Indicator 2 \\
IN3 & Limit signal 1 & OUT2 & Indicator 3 \\
IN4 & Limit signal 2 & OUT3 & Indicator 4 \\
\hline
\end{tabular}

\section{Summary}

The pneumatic manipulator is increasingly applied in the field of automation, this paper describes the design of a pneumatic manipulator based on the motion controller. Use the motion controller to save a lot of components, it can directly reduce the control cabinet volume, it will be a highly integrated system. The system has two types of mode, they are automatic and manual, you can choose according to the actual situation. The motion controller can improve the accuracy and reliability of the system, after the experiment, the manipulator operation is good, it has certain practical significance.

\section{References}

[1] Li Xiaoning. The development of pneumatic technology trend [J]. Mechanical manufacturing and automation, 2003, (2): 1-4.

[2] Zhao Tong. The development of pneumatic technology and application [J]. Hydraulic gas in the field of new and sealed, 2004, (2): 1-5.

[3] Bao Yanwei, Wu Yulan. A general pneumatic control design of manipulator [J]. Hydraulic and gas dynamic, 2005, (10): 82-83.

[4] Tian Ruoqiu. Stepper motor control system design based on PLC [J]. Digital technology and application, 2010, (2): 20-21.

[5] Xi Zhigang, Zhou Hongfu. The development and status of motion controller [J]. Electric drive automation, 2005, 32 (4): 5-10. 